\title{
CORRECTION
}

\section{Correction to: Screening delle tireopatie gestazionali, trattamento dell'ipotiroidismo subclinico o dell'ipotiroxinemia in gravidanza. Siamo ancora in cerca di risposte?}

\author{
Mariacarla Moleti ${ }^{1} \cdot$ Thomas Brix $^{2} \cdot$ Francesco Trimarchi $^{3} \cdot$ Laszlo Hegedüs $^{2}$
}

Pubblicato online: 19 dicembre 2017

(c) Springer International Publishing AG, part of Springer Nature 2017

\section{Correction to: L'Endocrinologo}

https://doi.org/10.1007/s40619-017-0377-0

Nel contributo originale, il nome di uno dei co-autori è stato erroneamente riportato. Il nome corretto è Laszlo Hegedüs. La pubblicazione originale è stata opportunamente corretta.

$凶$ F. Trimarchi

francesco.trimarchi@unime.it

1 Dipartimento di Medicina Clinica e Sperimentale, Università degli Studi di Messina, Messina, Italia

2 University of Southern Denmark, Odense, Denmark

3 Accademia Peloritana dei Pericolanti, Messina, Italia 